azithromycin or doxycycline for the treatment of rectal chlamydia. We investigated treatment efficacy of two treatments for rectal chlamydial infection.

Method A retrospective cohort of male and female patients diagnosed with rectal chlamydial infection between 2009 and 2015 was created at the STI services (Clinic 275) in Adelaide, Australia. Patients were included in the analysis if they were treated with azithromycin (1 g single dose) or doxycycline (100 mg twice a day for 10 days) and returned for repeat testing 14 to 180 days after treatment commenced. Log binomial models were used to estimate the relative risk (RR) of recurrent rectal chlamydia associated with the treatment with azithromycin versus doxycycline.

Results Of 526 patients diagnosed with rectal chlamydial infections over the study period, $73 \%(\mathrm{n}=384)$ were men and 27\% ( $\mathrm{n=142)}$ were women; $419(79.7 \%), 93(17.7 \%)$ and 14 $(2.6 \%)$ patients were treated with doxycycline, azithromycin or other medication respectively. Of these patients, 173 (41.3\%) of 419 doxycycline-treated patients and 31 (33.3\%) of 93 azithromycin-treated patients were retested between 14 and 180 days after treatment commenced $(p=0.16)$. Among these patients, the repeat rectal chlamydia test was less commonly positive in those treated with doxycycline $(5.8 \%$; $95 \%$ Confidence Interval (CI) $0.03-0.10)$ compared with those treated with azithromycin $(19.4 \%$; 95\% CI 0.09-0.36) and $(\mathrm{p}=0.01)$. In the multivariate analysis, azithromycin-treated patients had a significant higher risk of a positive test in the 14 and 180 days after treatment commenced (adjusted relative risk (aRR) 2.78, 95\% CI 1.10-7.05).

Conclusion The findings suggest that doxycycline may be more effective than azithromycin in treating rectal chlamydial infection.

\section{P3.27 LYMPHOGRANULOMA VENEREUM IN SWEDEN 2004- 2016: INCREASED RATES AMONG HIV-NEGATIVE MEN WHO HAVE SEX WITH MEN AND CHANGED GENOTYPES}

${ }^{1}$ Björn Herrmann, ${ }^{1}$ Jenny Isaksson, ${ }^{1}$ Ola Carlsson, ${ }^{2}$ Åsa Airell, ${ }^{1}$ Susanne Strömdahl, ${ }^{3}$ Göran Bratt. 'Uppsala University, Uppsala - Sweden; ${ }^{2}$ Karolinska University Hospital, Stockholm - Sweden; ${ }^{3}$ South General Hospital, Stockholm - Sweden

\subsection{6/sextrans-2017-053264.264}

Introduction Lymphogranuloma venereum (LGV) has become prevalent among men who have sex with men (MSM) in Europe since an outbreak in The Netherlands in 2003. The aim of this study was to describe the development of LGV in relation to HIV since 2004, and genotypes of LGV and other Chlamydia trachomatis $(\mathrm{Ct})$ genovars in a MSM population in 2014/15.

Methods All testing for LGV in Sweden is referred to Uppsala University Hospital. LGV-specific pmpH-gene PCR was used for detection. High-resolution genotyping based on ompA gene and multilocus sequence typing (MLST) was performed on all Ct-positive cases from an STI clinic for MSM in Stockholm between 1.9. 2014 and 1.7.2015.

Results The annual numbers of detected LGV cases in Sweden were 2 in 2004 to 2006, between 5 and 20 in 2007 to 2012, and between 23 and 38 in 2013 to 2016. The number of LGV-tests increased from 68 in 2008 to 268 in 2016. During the study in 2014/15 31 LGV cases were identified in 309 patients with successful PCR-results. In 39\% (12/31) LGV was unexpected and had not been detected without extended screening. The HIV-prevalence among LGV-positive patients decreased from $88 \% 2006-2013 \%$ to $68 \%$ 2014-2015. Of ompA genotyped LGV cases $69 \%$ were L2\% and $31 \%$ were L2b type. This contrasts to earlier Swedish and European data from 2004-2009 when only L2b was found. Complete genotyping, including ompA and MLST, was obtained for 151 patients with non-LGV Ct and resulted in genovar D, 27\%; E, 14\%; G, 30\% and J 21\%. MLST resulted in 27 STs of which 3 predominated and accounted for 51\%. Eight STs were new when compared to the database http://mlstdb.bmc. uu.se comprising 540 STs from $>3300$ specimens.

Conclusion In Sweden LGV has gone from sporadic import cases to a probable endemic spread among HIV-negative MSM, which emphasises the need for LGV-testing This emphasises the need for LGV-testing within this high risk group. LGV has developed from being of clonal nature to occur as different strains among MSM.

\section{P3.28 IMPROVING THE EVIDENCE-BASE TO UNDERSTAND STI RISK REDUCTION CAPACITY: THE FEASIBILITY AND ACCEPTABILITY OF LINKING ONLINE BEHAVIOURAL SURVEY DATA TO ELECTRONIC PATIENT RECORDS}

\begin{abstract}
${ }^{1,2,3} \mathrm{P}$ Blomquist, ${ }^{1,2} \mathrm{~S}$ Wayal, Cath Mercer, ${ }^{1,2,4} \mathrm{D}$ Reid, ${ }^{1,2,4} \mathrm{P}$ Weatherburn, ${ }^{1,2,3} \mathrm{G}$ Hughes ${ }^{1}$ National Institute of Health Research (NIHR) Health Protection Research Unit (HPRU) in Blood Borne and Sexually Transmitted Infections; ${ }^{2}$ Centre for Sexual Health and HIV, Research Department of Infection and Population Health, University College London; 3 London School of Hygiene and Tropical Medicine, London; ${ }^{4}$ Public Health UK
\end{abstract}

\subsection{6/sextrans-2017-053264.265}

Introduction Behavioural surveys can provide insight into the attitudes and context associated with risk of sexually transmitted infections (STIs), but interpretation is hampered by theirreliance on self-reported STI history rather than confirmed diagnoses. We aimed to determine the feasibility and acceptability of linking clinic patients' online survey data on STI risk factors with the national surveillance dataset on STI diagnoses (GUMCADv2), which contains electronic patient record (EPR) data routinely submitted to Public Health England by sexual health clinics.

Methods Between May and September 2016, attendeesat 16 sexual health clinicsacross Englandwereinvited to completean onlinesurveyon knowledge, attitudes, and behaviours around STI risk, using a clinic tablet or personal device (e.g. smartphone). Participants were given a unique study identifier (ID) to type into the survey, and provided consent to participate and to data linkage. Screening questions routed eligible participants, $\geq 15$ years old and sexually active in the past year, to the full survey. Recruiting clinic staff kept record of study IDs and corresponding patient IDs. We linked surveysto GUMCADv2 with deterministic and probabilistic methods, using the recorded ID numbers as well as age, gender, and clinic attendance date. We examined recruitment and linkage success for a target of 7500 eligible attendees, and used univariable logistic regression and Z-test for proportions to assess selection bias.

Results 6283 clinic attendees agreed to take part in the study, of whom73.6\% (4626) completed the survey. 95.9\% (4437) of survey respondents were eligible; $59.2 \%$ of our recruitment target. Survey completionamong those agreeing to participate was higher in the 9 clinics that recruited $\geq 50 \%$ of their target than those that did not $(84.9 \%$ vs $53.0 \%)$. Survey completion was also higher in those who agreed to fill in the survey in clinic than in those who agreed to do soat home $(87.3 \%$ vs 